\title{
A mathematical model for SARS-CoV-2 in variable-order fractional derivative
}

\author{
Mahmoud H. DarAssi ${ }^{1}$, Mohammad A. Safi ${ }^{2}$, Muhammad Altaf Khan ${ }^{3, a} \mathbb{D}$, Alireza Beigi ${ }^{4}$, Ayman A. Aly $^{5}$, and \\ Mohammad Y. Alshahrani ${ }^{6}$ \\ 1 Department of Basic Sciences, Princess Sumaya University for Technology, Amman 11941, Jordan \\ 2 Department of Mathematics Faculty of science, The Hashemite University, P. O. Box 330127, Zarqa 13133, Jordan \\ 3 Institute for Ground Water Studies, Faculty of Natural and Agricultural Sciences, University of the Free State, \\ Bloemfontein, South Africa \\ 4 School of Mechatronic Systems Engineering, Simon Fraser University, 102 Avenue, Surrey, BC V3T 0A3 250-13450, \\ Canada \\ ${ }^{5}$ Department of Mechanical Engineering, College of Engineering, Taif University, P.O.Box 11099, Taif 21944, \\ Saudi Arabia \\ 6 Department of Clinical Laboratory Sciences, College of Applied Medical Sciences, King Khalid University, P.O. Box 61413, \\ Abha 9088, Saudi Arabia
}

Received 2 November 2021 / Accepted 13 January 2022 / Published online 3 February 2022

(C) The Author(s), under exclusive licence to EDP Sciences, Springer-Verlag GmbH Germany, part of Springer Nature 2022

\begin{abstract}
A new coronavirus mathematical with hospitalization is considered with the consideration of the real cases from March 06, 2021 till the end of April 30, 2021. The essential mathematical results for the model are presented. We show the model stability when $\mathcal{R}_{0}<1$ in the absence of infection. We show that the system is stable locally asymptotically when $\mathcal{R}_{0}<1$ at infection free state. We also show that the system is globally asymptotically stable in the disease absence when $\mathcal{R}_{0}<1$. Data have been used to fit accurately to the model and found the estimated basic reproduction number to be $\mathcal{R}_{0}=1.2036$. Some graphical results for the effective parameters are drawn for the disease elimination. In addition, a variable-order model is introduced, and so as to handle the outbreak effectively and efficiently, a genetic algorithm is used to produce high-quality control. Numerical simulations clearly show that decision-makers may develop helpful and practical strategies to manage future waves by implementing optimum policies.
\end{abstract}

\section{Introduction}

The corona virus affected and effecting the populations throughput the world by providing severe infection and death. The early and effective strategy to overcome this infection needed to follow the rule and regulations suggested by the World health organization to prevent the people from further reinfection. It is well known that every researchers from their respective field of research provided great efforts for the elimination of this infection. Many countries were the infection provided many deaths with so many waves of infection. Pakistan is one of among those where there are some waves occurred for this COVID-19 infection from which the population suffered. It has been reported the number of reported cases and deaths in each wave and seems the increase in the cases. There has been reported the number of death percentage in wave 1 and 2 to be $1 \%$.

The formulation of mathematical models in epidemiology and engineering areas has proven his role to be important; see [1-3]. Many researchers in different per-

\footnotetext{
a e-mail: altafdir@gmail.com (corresponding author)
}

spective studied the coronavirus infection. While the mathematicians and biologists who worked on the modeling of the COVID-19 infection studied and formulated the mathematical models with real cases of different countries and presented about the peak of the infection and its eliminations of infection. In this regard, some mathematical models are highlighted here that formulated for this infection are [4-10]. The initial cases reported in China for the COVID-19 infection have been investigated in [4] through a mathematical model. The real cases from Spain and Italy are considered in the modeling of infection in the form of an SEIR model which has been studied in [5]. The COVID-19 infection dynamics and its prediction through a mathematical model of an SEIR have been investigated in [6]. The data from Italy are considered, and a mathematical model is formulated for the COVID-19 infection and its analysis has been considered in [7]. The impact of social distancing and other features that can be considered essential for the decrease in the COVID-19 infection has been studied by the authors in [8]. They formulated a mathematical model for the South African infected cases. A comparison study for the dynamics 
of coronavirus infection has been discussed in [9]. The number of real cases from Mexico population through a mathematical model is considered in [10]. The real infected cases from KSA are considered by the authors using mathematical modeling approach and obtained the results regarding the disease eliminations in the country [11]. The modeling and the contrail measure of infection of COVID-19 have been discussed in [12]. The disease eliminations and its control through optimal control theory are suggested by the authors in [13]. Addressing the model with lockdown measure for the COVID-19 infection is discussed in [14]. Some other interesting work related to the modeling of COVID19 and related results regarding the disease are suggested in [15-17]. Moreover, the authors in [18-22] considered the COVID-19 infection models in fractional derivatives and some recommendations regarding the minimization of infection are given in the form of lockdown and control strategies. Moreover, the applications of fractional calculus and the numerical methods for the physical and biological problems have been discussed in [23-26,29-39]. For example, the HIV infection model with antiviral drug therapy is considered in [23]. A fractional SEIR model using wavelet method has been proposed in [24]. Some biological models with analytical and numerical techniques are discussed in [25]. A fractional model in ABC operator in astrophysics is discussed in [26]. A recent study is conducted for the understanding of coronavirus infection and its controls [27]. A mathematical model in non-singular order to investigate the coronavirus infection is considered in [28]. Nonetheless, some studies show that in some conditions, time-varying fractional-order models are able to better demonstrate behavior of systems [40-47]. However, now, there are few studies on time-varying fraction diseases dynamics, and it demands more studies in this field. Motivated by this in the current research, we propose and study a variable-order fractional model of SARS-CoV-2 dynamic.

In this work, a new mathematical model in the presence of hospitalization class is considered to better understand the COVID-19 infection in the presence of hospitalization and then using the concept of variable fractional order to study its dynamics. First, we will study the model in integer order and study the detailed mathematical results, and then, we extend the proposed model to have the results for the variable fractionalorder model. We discuss in details the formulation of the model in Sect. 2. The stability results and the model endemic equilibria have been shown in Sect. 3. Estimations of the model parameters and its numerical results are discussed briefly in Sect. 4 and 5, respectively. In Sect. 6, using the approach of variable order and its related results are discussed. All the results are summarized and concluded in Sect. 7.

\section{Model formulation}

Due to the COVID infection and its emerging in the society that producing many cases again and again in the society and creates a lot of difficulties for the humans as well as the government. Therefore, the eradications of the infections and to prevent the populations further, government present policies in light to World health organization. Therefore, here, our aims by formulating a novel mathematical model in the presence of hospitalization infection to understand in an effective way the dynamics of the COVID-19 infection with real cases reported from Pakistan. We denote the population of humans by $N(t)$ and split into it further into six compartments: The susceptible $S(t)$, exposed $E(t)$, infected with visible symptoms $I(t)$, infected with no visible symptoms $A(t)$, the individuals that are hospitalized/ quarantined $H(t)$ and those recovered from infection is $R(t)$. We present the model below in the form of differential equations based on the above discussions

$$
\left\{\begin{array}{l}
\frac{d S}{d t}=\Pi-\rho(I+\sigma A) \frac{S}{N}-\omega S \\
\frac{d E}{d t}=\rho(I+\sigma A) \frac{S}{N}-\left(\psi+\omega+\omega_{0}\right) E \\
\frac{d I}{d t}=\psi(1-\tau) E-\left(\omega+\omega_{1}+\gamma_{1}+\nu\right) I \\
\frac{d A}{d t}=\psi \tau E-\left(\omega+\omega_{2}+\gamma_{2}\right) A \\
\frac{d H}{d t}=\nu I-\gamma_{3} H-\omega_{3} H-\omega H \\
\frac{d R}{d t}=\gamma_{1} I+\gamma_{2} A+\gamma_{3} H-\omega R
\end{array}\right.
$$

subject to the initial conditions that are non-negative. The parameters given in the model (1) are defined as: The populations of healthy people that are susceptible to get corona virus infection are generated through the birth rate $\Pi$, while the natural death rate for each compartment in the model is shown by $\omega$. The contact rate that generates the infection is given by $\rho$, while the infection that generated through the individuals with no clinical symptoms is given by the parameter $\sigma$. The death due to disease in the compartments of $E, I, A$, and $H$ is shown by $\omega_{0}, \omega_{1}, \omega_{2}$, and $\omega_{3}$. The parameters $\psi$ and $\tau$ distribute the infection in symptomatic and asymptomatic. The infected people are hospitalized at the rate $\nu$. The recovery rate from the infected, infected with no clinical symptoms, and hospitalized people is shown by $\gamma_{i}$ for $i=1,2,3$, respectively. We write the model (2) in the absence of the last equation, given by

$$
\left\{\begin{array}{l}
\frac{d S}{d t}=\Pi-\rho(I+\sigma A) \frac{S}{N}-\omega S \\
\frac{d E}{d t}=\rho(I+\sigma A) \frac{S}{N}-\left(\psi+\omega+\omega_{0}\right) E \\
\frac{d I}{d t}=\psi(1-\tau) E-\left(\omega+\omega_{1}+\gamma_{1}+\nu\right) I \\
\frac{d A}{d t}=\psi \tau E-\left(\omega+\omega_{2}+\gamma_{2}\right) A \\
\frac{d H}{d t}=\nu I-\left(\gamma_{3}+\omega_{3}+\omega\right) H
\end{array}\right.
$$

We have the total dynamics for the system (2) shown by

$$
\frac{d N}{d t}=\Pi-\omega N-\omega_{0} E-\omega_{1} I-\omega_{2} A-\omega_{3} H \leq \Pi-\omega N .
$$


Thus

$$
\frac{d N}{d t} \leq \Pi-\omega N
$$

and further

$$
N(t) \leq \frac{\Pi}{\omega} \text { whenever } \mathrm{t} \longrightarrow \infty .
$$

We consider the region given below for the system (2)

$$
\Gamma=\left\{(S, E, I, A, H) \in \mathbb{R}_{+}^{5}: N(t) \leq \frac{\Pi}{\omega}\right\},
$$

which is feasible biologically and all the solutions contained in it.

\subsection{Equilibria}

The equilibria for the system (2) in case if there is no infection in the population denoted by $E_{0}$ are shown below

$$
D_{0}=\left(S^{0}, 0,0,0,0\right)=\left(\frac{\Pi}{\omega}, 0,0,0,0\right) .
$$

Next, we compute the basic reproduction number $\mathcal{R}_{0}$, using analysis given in [48], and present the results given by

$$
F=\left(\begin{array}{cccc}
0 & \rho & \tau & \rho \\
0 & 0 & 0 & 0 \\
0 & 0 & 0 & 0 \\
0 & 0 & 0 & 0
\end{array}\right), \quad V=\left(\begin{array}{cccc}
l_{1} & 0 & 0 & 0 \\
-\psi(1-\tau) & l_{2} & 0 & 0 \\
-\psi \tau & 0 & l_{3} & 0 \\
0 & -\nu & 0 & l_{4}
\end{array}\right)
$$

where $l_{1}=\left(\psi+\omega+\omega_{0}\right), l_{2}=\left(\omega+\omega_{1}+\gamma_{1}+\nu\right), l_{3}=$ $\left(\omega+\omega_{2}+\gamma_{2}\right)$, and $l_{4}=\left(\gamma_{3}+\omega_{3}+\omega\right)$. The following expression is the required basic reproduction number for our considered system and is given by

$$
\mathcal{R}_{0}=\frac{l_{2} \rho \sigma \tau \psi+l_{3} \rho(1-\tau) \psi}{l_{1} l_{2} l_{3}} .
$$

\section{Stability results}

In the following, the stability analysis for the considered system (2) at the disease-free equilibrium $E_{0}$ is carried out.

Theorem 1 The system (2) at the disease-free case $E_{0}$ is locally asymptotically stable whenever $\mathcal{R}_{0}<1$.
Proof Computing the Jacobian at the disease-free case $E_{0}$ is given by

$$
J\left(E_{0}\right)=\left(\begin{array}{ccccc}
-\omega & 0 & -\rho & -\rho \sigma & 0 \\
0 & -l_{1} & \rho & \rho \sigma & 0 \\
0 & (1-\tau) \psi & -l_{2} & 0 & 0 \\
0 & \tau \psi & 0 & -l_{3} & 0 \\
0 & 0 & \nu & 0 & -l_{4}
\end{array}\right) .
$$

We get easily the two roots $-\omega$ and $-l_{4}$. The remaining eigenvalues containing negative real parts can be determined through the equations given by

$$
\lambda^{3}+f_{1} \lambda^{2}+f_{2} \lambda+f_{3}=0,
$$

where

$$
\begin{aligned}
& f_{1}=l_{1}+l_{2}+l_{3}, \\
& f_{2}=l_{2} l_{3}+l_{1}\left(l_{2}+l_{3}\right)+\rho \psi(-\sigma \tau+\tau-1), \\
& f_{3}=l_{1} l_{2} l_{3}\left(1-\mathcal{R}_{0}\right) .
\end{aligned}
$$

It can be observed that $f_{i}$ for $i=1,2,3$ are positive whenever $\mathcal{R}_{0}<1$, and furthermore, it can be obtained easily the conditions of Routh-Hurtwiz criteria, $f_{i}>0$ and $f_{1} f_{2}-f_{3}>0$, for $i=1,2,3$. Therefore, it can be concluded that the system given in (2) at $E_{0}$ when $\mathcal{R}_{0}<1$ is locally asymptotically stable.

We state and prove the following theorem to show that the system (2) at $E_{0}$ is globally asymptotically stable.

Theorem 2 The system (2) at $E_{0}$ is locally asymptotically if $\mathcal{R}_{0} \leq 1$.

Proof Define the Lyapunov function given by

$$
\mathcal{L}(t)=\xi_{1} E(t)+\xi_{2} I(t)+\xi_{3} A(t),
$$

where $\xi_{m}>0$ for $m=1,2,3,4$, that can be adjusted their values at later stage. Taking $\frac{d}{d t}$ of $\mathcal{L}$, we have

$$
\mathcal{L}^{\prime}(t)=\xi_{1} E^{\prime}+\xi_{2} I^{\prime}+\xi_{3} A^{\prime} .
$$

Using the equations from (2) and making some setting, we have

$$
\begin{aligned}
\mathcal{L}^{\prime}(t)= & \xi_{1}\left[\rho(I+\sigma A) \frac{S}{N}-l_{1} E\right] \\
& +\xi_{2}\left[\psi(1-\tau) E-l_{2} I\right]+\xi_{3}\left[\psi \tau E-l_{3} A\right], \\
= & {\left[\xi_{2} \psi(1-\tau)+\xi_{3} \psi \tau-l_{1} \xi_{1}\right] E+\left[l_{1} \rho-l_{2} \xi_{2}\right] I } \\
& +\left[\xi_{1} \sigma \rho-l_{3} \xi_{3}\right] A .
\end{aligned}
$$

Let us consider the values for the constants $\xi_{1}, \xi_{2}$, and $\xi_{3}$. We choose as follows: $\xi_{1}=l_{2}, \xi_{3}=\rho$, and $\xi_{3}=$ $\rho \sigma l_{2} / l_{3}$. Then, we have the following results finally: 


$$
\mathcal{L}^{\prime}(t)=-l_{1} l_{2}\left[1-\mathcal{R}_{0}\right] E
$$

Here, $\mathcal{R}_{0}<1$, and so, we have $\mathcal{L}^{\prime}(t)$ is negative and so the model (2) is globally asymptotically stable at $E_{0}$.

\subsection{Endemic equilibria}

Here, we present and obtain the endemic equilibria of the system (2) by describing its endemic equilibrium by $E_{1}=\left(S^{*}, E^{*}, I^{*}, A^{*}, H^{*}\right)$, and so, we have the below

$$
\left\{\begin{array}{l}
S^{*}=\frac{\Pi}{\lambda^{*}+\omega} \\
E^{*}=\frac{\lambda^{*} S^{*}}{l_{1}} \\
I^{*}=\frac{(1-\tau) \psi E^{*}}{l_{2}} \\
A^{*}=\frac{e \tau \psi}{l_{3}}, \\
R^{*}=\frac{\nu I^{*}}{l_{4}} .
\end{array}\right.
$$

Consider the above values in the following equation:

$$
\lambda^{*}=\frac{\rho\left(I^{*}+\tau A^{*}\right)}{N^{*}},
$$

getting the following:

$$
\Phi_{1} I^{*}+\Phi_{2}=0
$$

where

$$
\begin{aligned}
& \Phi_{1}=l_{3}(1-\tau) \psi\left(l_{4}+\nu\right)+l_{2} l_{4}\left(l_{3}+\tau \psi\right), \\
& \Phi_{2}=l_{1} l_{2} l_{3} l_{4}\left(1-\mathcal{R}_{0}\right) .
\end{aligned}
$$

We can see that $\Phi_{1}>0$, while $\Phi_{2}$ can be positive depending on the value of $\mathcal{R}_{0}$. When $\mathcal{R}_{0}<1$, so we getting $I^{*}=-\Phi_{2} / \Phi_{1}$, and thus, there is no equilibria. The requirements for the positive endemic equilibria are to have $\mathcal{R}_{0}>1$, and hence, for the system (2), a unique positive endemic equilibrium exists.

\section{Estimations of the parameters}

To understand effectively the dynamics of an epidemic model, it is necessary or useful to have the real data to demonstrate the model parameters and to obtain reasonable results for the epidemic model. In this regard, we consider the cases from March 06, 2021, till April 30, 2021 for the finding of parameter values; we can refer the readers for the real data [49]. The population of Pakistan considered here is $N(0)=220000000$, and we estimate the parameters $\Pi$ and $\omega$ as follows: $\Pi \approx 8903$ per day using the expression $N(0)=\Pi / \omega$, and $\omega=1 /(67.7 \times 365)$, where $\omega=1 /(67.7)$ is the average life span in Pakistan. The parameters other than these can be obtained through the model data fitting. The data considered in this paper from March 06, 2021to April 30, 2021. We use the curve fitting technique to get the values of the parameters, as shown in Table 1, and the possible fitting of the model to the data is shown in Fig. 1a with the estimated basic reproduction number $\mathcal{R}_{0} \approx 1.2036$. Figure $1 \mathrm{~b}$ describes the behavior of the model versus data for long time level. It follows from Fig. 1(a) that the model behaves good to the real cases. Thus, the model parameters considered in Table 1 are realistic and can better study the dynamics of the COVID-19 infection.

\section{Numerical results}

Here, we consider the system (2) with the consideration of the cases reported from March to the end of April in Pakistan to understand the parameters impact on the model. The cases consider here are in daily basis, so the time unit is to be per day. In numerical results, we consider the parameters values shown in Table 1. We

Table 1 Estimated parameters

\begin{tabular}{llll}
\hline Symbol & Definition & Value/per day & Source \\
\hline$\Pi$ & Recruitment rate & $\omega \times N(0)$ & 1 \\
$\omega$ & Natural death rate & $\frac{1}{67.7 \times 365}$ & Estimated \\
$\rho$ & Disease contact rate & 0.9549 & {$[50]$} \\
$\sigma$ & Disease contact of A & 0.9635 & {$[51]$} \\
$\psi$ & Incubation period & 0.7961 & {$[51]$} \\
$\omega_{0}$ & Disease death rate of exposed people & 0.0126 & Fitted \\
$\omega_{3}$ & Death due to infection at $H$ & 0.9635 & {$[51]$} \\
$\tau$ & Progress to asymptomatic infection & 0.0010 & Fitted \\
$\omega_{1}$ & Death due to infection at $I$ & 0.1456 & Fitted \\
$\gamma_{1}$ & Recovery of symptomatic people & 0.0069 & {$[51]$} \\
$\omega_{2}$ & Disease death of asymptomatic people & {$[51]$} & {$[51]$} \\
$\gamma_{2}$ & Recovery of asymptomatic people & 0.8666 & \\
$\nu$ & Hospitalization rate of symptomatic people & 0.021 & Fitted \\
\hline
\end{tabular}




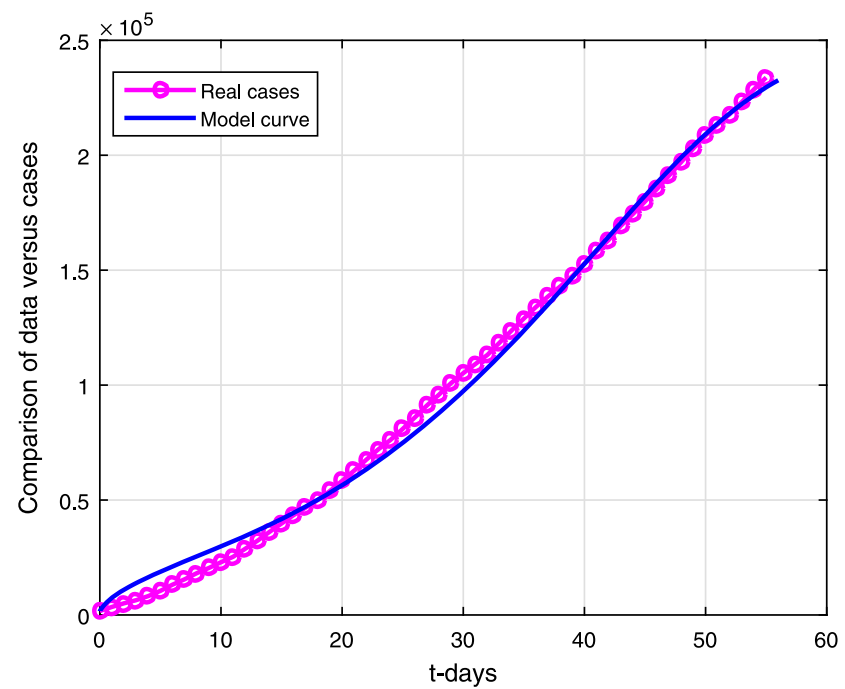

(a)

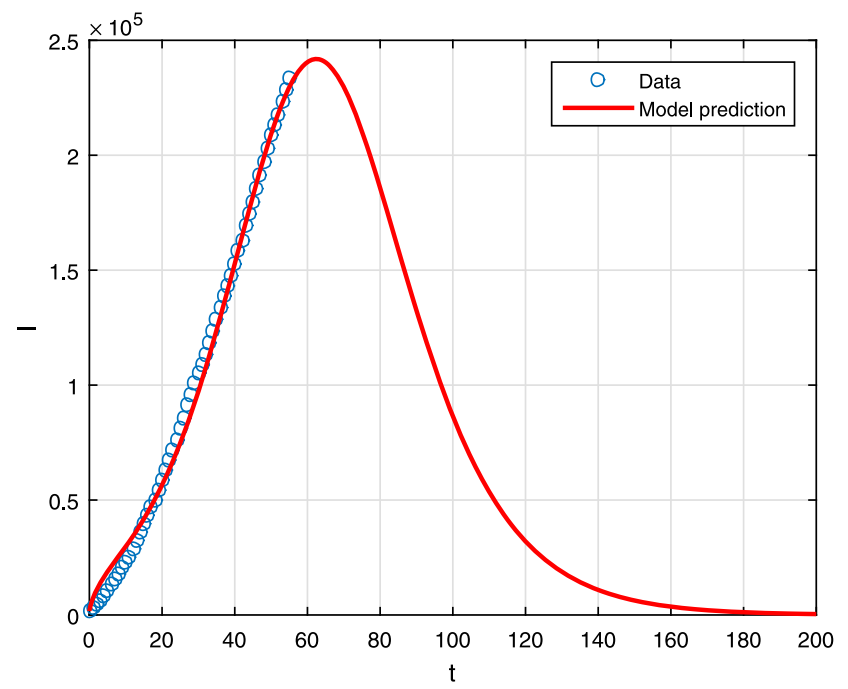

(b)

Fig. 1 a Reported cases versus model prediction. b Long time behavior of model versus cases

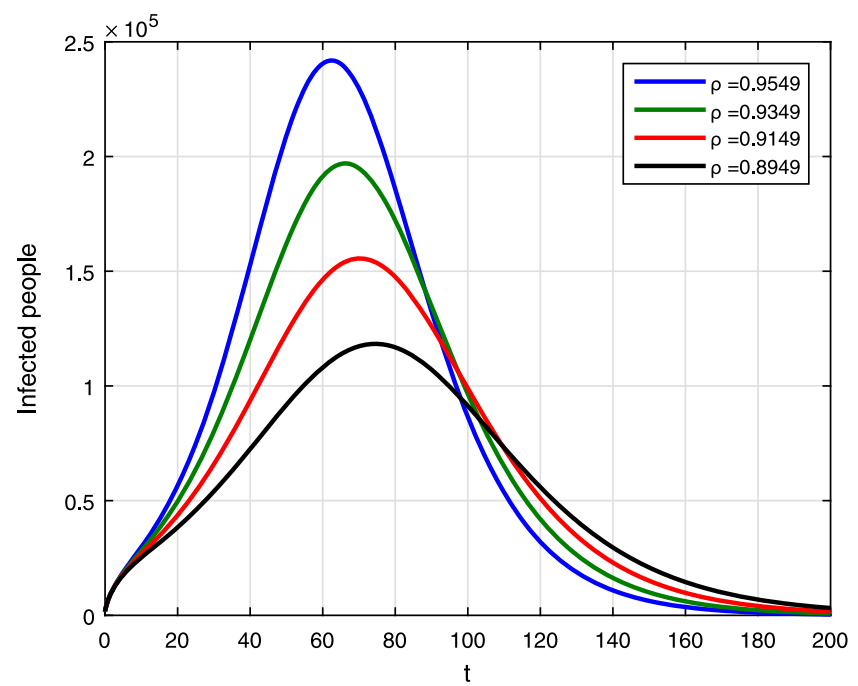

(a)

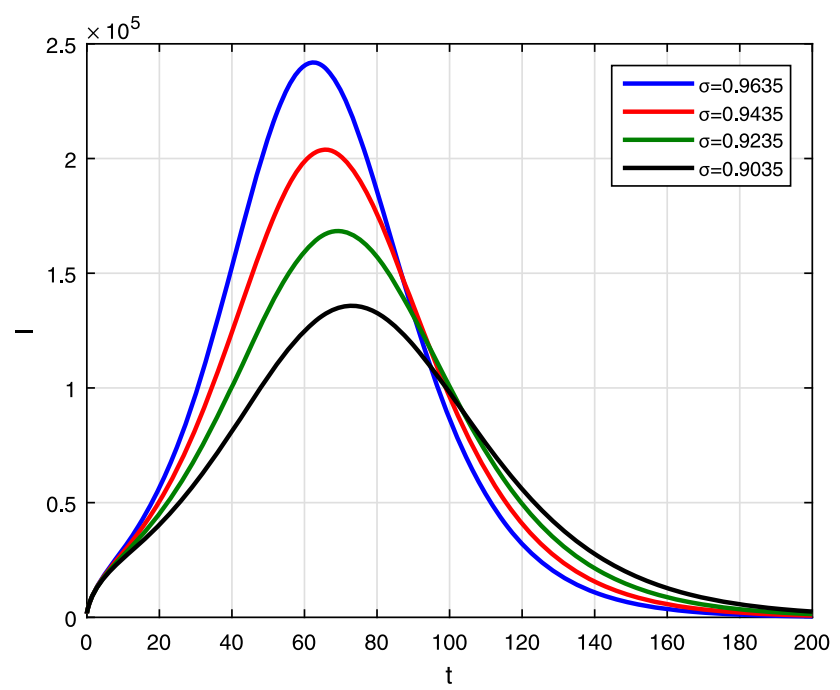

(b)

Fig. 2 a $\rho$ with different values; $\mathbf{b} \sigma$ with different values

estimate the initial values of the model (2) is as follows: $S(0)=219698286, E(0)=300000, I(0)=1714$, while assuming that that there is no recovery from the infection yet and so $R(0)=0$, and no enough information about the asymptomatic and hospitalized data, so we consider $A(0)=H(0)=0$. We consider the disease contact rate $\rho$ and $\sigma$ with different values on the dynamics of infected people shown in Fig. 2. In Fig. 2a, we see when decreasing the value of $\rho$, the number of infected people decreases faster. It can concluded that the prevention from the infection using the instructions suggested by World Health organization should be follows to decrease the disease burden from the populations. Washing hands, social distances, using masks, avoid gathering, self-quarantine, and restrictions to home can better reduce the infection. In Fig. 2b, one can see that the infection decreases when decreasing the value of $\sigma$. The parameter $\sigma$ that denote the contacts of healthy people with asymptomatic infected people (individuals do now show clinical symptoms) can decrease the infection risk in the population. It should be noted that asymptomatic infected does not show visible disease symptoms and can increase the infection. It is documented that the number of infected individuals due to COVID-19 has high percentage of infection due to asymptomatic infection. Therefore, it is important for the government to make increase the testing facility to identify the number of asymptomatic infected people. The impact of the parameters $\psi, \tau$ and $\nu$ has been shown graphically in Fig. 3. Decreasing the values of these parameters, we have decrease in the number of infected people. 


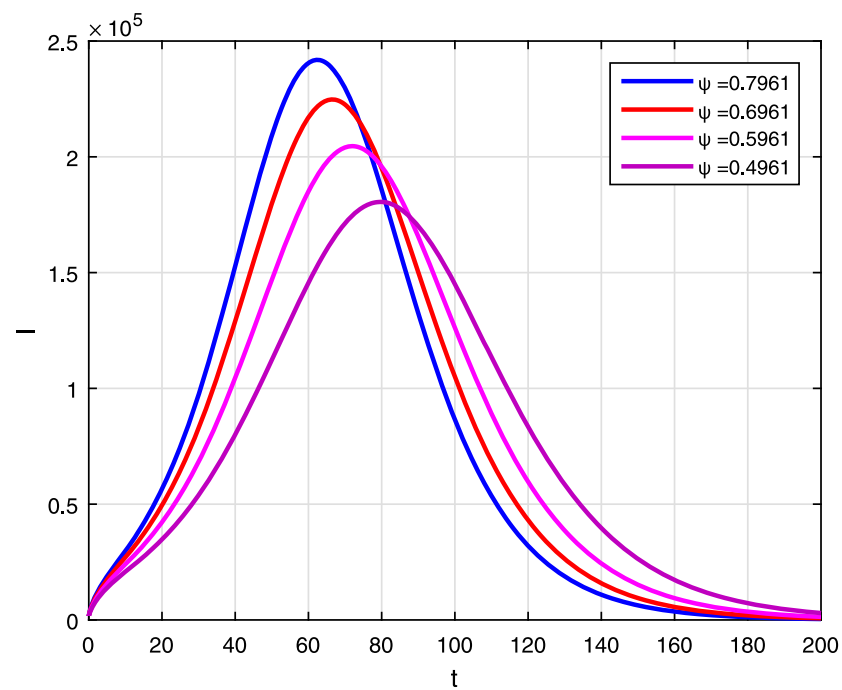

(a)

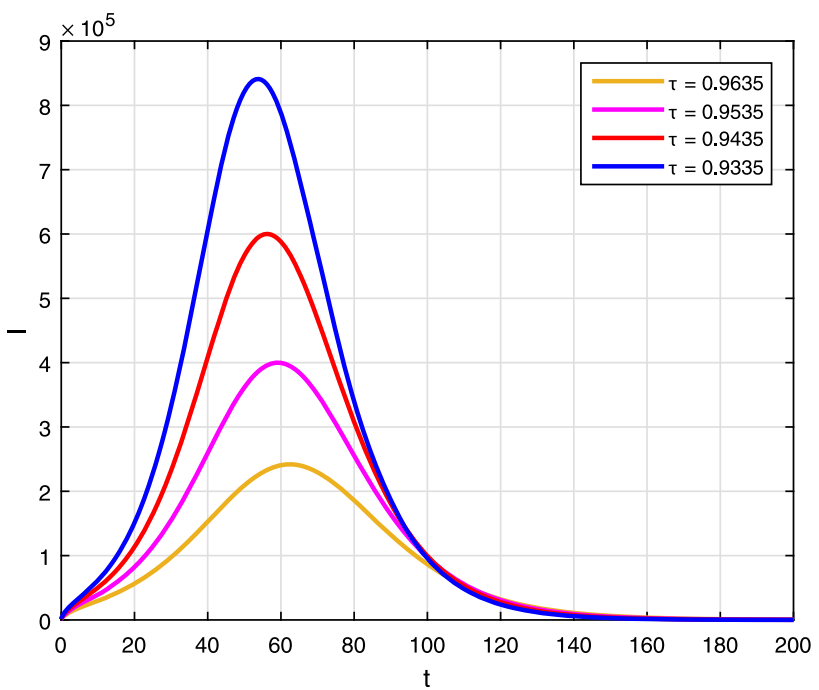

(b)

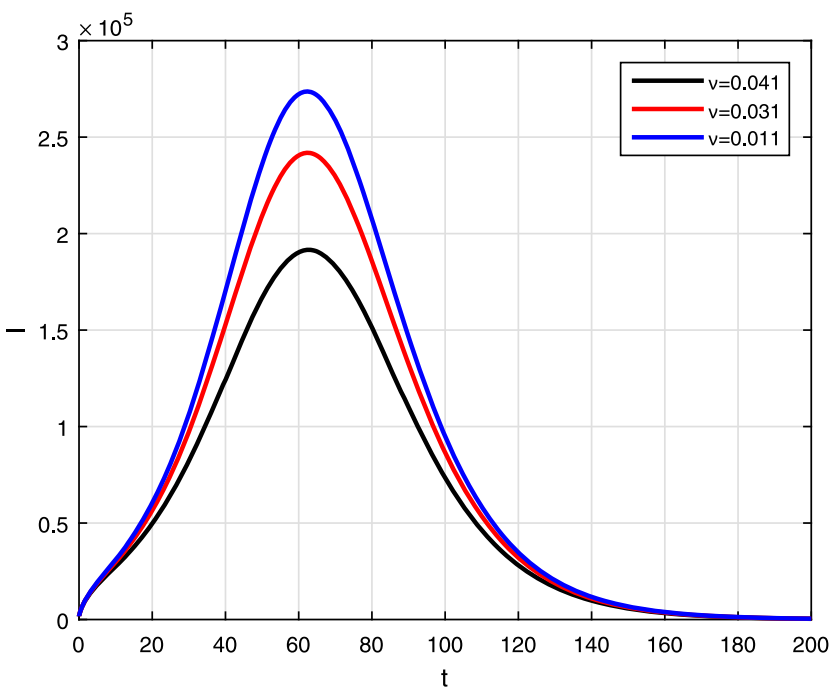

(c)

Fig. 3 a $\psi$ with different values, $\mathbf{b} \tau$ with different values, and $\mathbf{c} \tau$ with different values

\section{Variable-order fractional model of SARS-CoV-2 dynamic}

It has been shown in the literature that fractional calculus is able to produce more accurate results in modeling natural systems and processes. As a result, in this part, we present a novel variable-order fractional model of SARS-CoV-2 dynamics in Pakistan. Also, we consider an additional component, namely, hospitalized people $(\mathrm{H})$. The fractional model with variable order is given as follows:

$$
\begin{aligned}
& { }_{0}^{c} D_{t}^{q(t)} S=\Pi-\rho(I+\sigma A) \frac{S}{N}-\omega S \\
& { }_{0}^{c} D_{t}^{q(t)} E=\rho(I+\sigma A) \frac{S}{N}-\left(\psi+\omega+\omega_{0}\right) E \\
& { }_{0}^{c} D_{t}^{q(t)} I=\psi(1-\tau) E-\left(\omega+\omega_{1}+\gamma_{1}+\nu\right) I
\end{aligned}
$$

$$
\begin{aligned}
& { }_{0}^{c} D_{t}^{q(t)} A=\psi \tau E-\left(\omega+\omega_{2}+\gamma_{2}\right) A \\
& { }_{0}^{c} D_{t}^{q(t)} H=\nu I-\left(\gamma_{3}+\omega_{3}+\omega\right) H \\
& { }_{0}^{c} D_{t}^{q(t)} H=\gamma_{1} I+\gamma_{2} A+\gamma_{3} H-\omega R .
\end{aligned}
$$

$\gamma_{H}$ is the recovery rate of quarantined infected individuals and $\delta_{I}$ is the rate of symptomatic infected individual's transition to the quarantined infected component. Also, $q(t)$ indicates the time-varying fractional-order derivative. In this study, the Caputo fractional derivative based on the predictor-corrector method is applied for numerical calculations of the fractional model. It is one of the most widely used methods for analyzing fractional differential equations in a chaotic manner (see $[52,53]$ for more details on the predictor-corrector method). To investigate the effects of the time-varying fractional derivative, we present the results of the model with two different fractional derivatives. Figure 4 shows 
Fig. 4 The number of individuals when $q_{1}(t)=0.9$ and $q(t)=0.9+\frac{0.1}{1+\exp (-t)}$
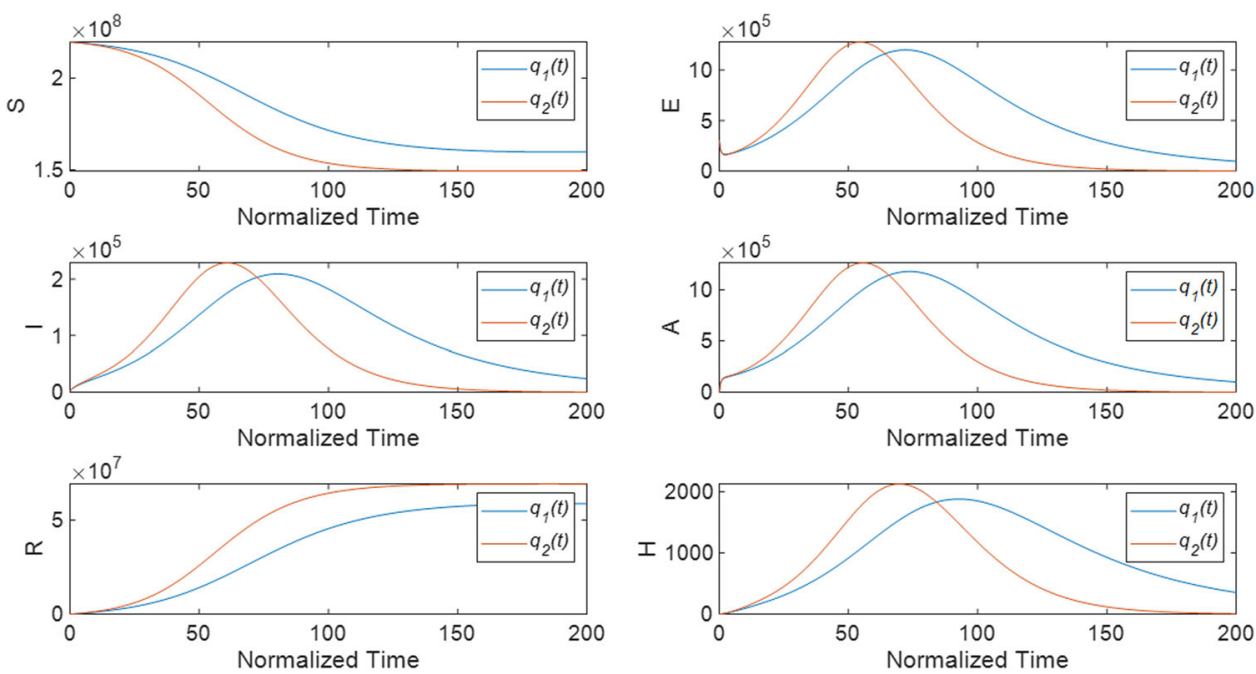

Table 2 Parameters of genetic algorithm

\begin{tabular}{ll}
\hline Parameter & Value \\
\hline Crossover fraction & 0.75 \\
Population size & 90 \\
Selection function & Tournament \\
Mutation function & Constraint-dependent \\
Crossover function & Intermediate \\
Migration direction & Forward \\
Migration fraction & 0.3 \\
Migration interval & 30 \\
Stopping criteria & 40000 \\
\hline
\end{tabular}

the number of individuals when $q_{1}(t)=0.9$ and $q(t)=$ $0.9+\frac{0.1}{1+\exp (-t)}$. As it is evident from this figure, when the value of fractional derivative varies by time, the results are different, and it can dramatically affect the behavior of the model.

\subsection{Optimal decision rules}

Herein, we propose a genetic optimization approach to find optimal decision rules. Genetic optimization is an appropriate technique to handle such problems, since there is a trade-off between all possible values. Table 3 lists the parameters and configuration of the applied multi-objective genetic algorithm. The fractional-order derivative is considered to vary over time as $q(t)=0.9+$ $\frac{0.1}{t+1}$.

We consider $\delta_{I}$ as the decision variable. The following is the cost functions that have been chosen:

$$
J=\sum E(t)+I(t)+A(t)
$$

We have chosen this cost function to reduce the accumulated infected people. In what follows the results of the optimization are provided.

Figure 5 shows the value of the cost function from the genetic algorithm optimization. As it is shown in this figure, we could choose the optimal controllers, which

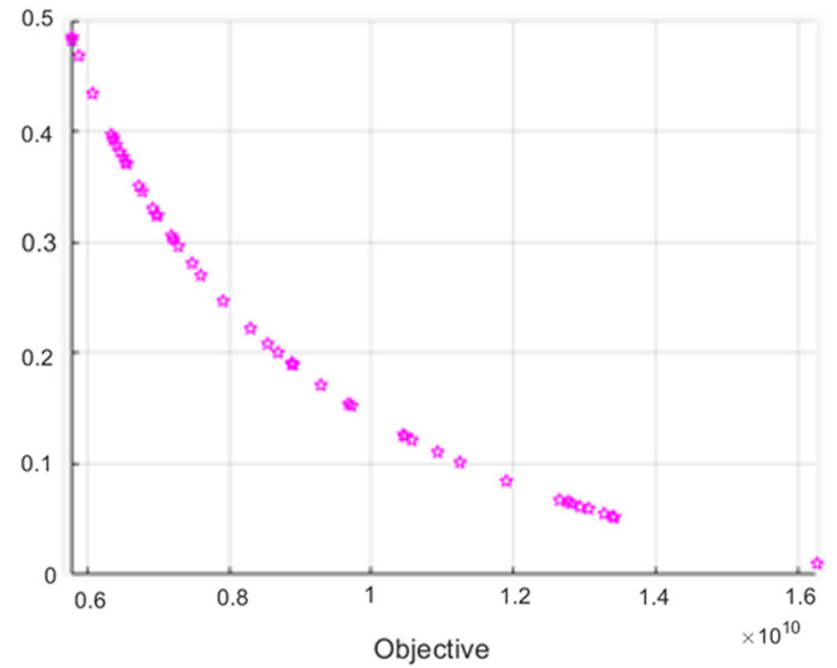

Fig. 5 Objective function

effectively reduce both cost functions. Herein, we chose $\delta_{I}=0.18$ as the optimal value for the control purpose. The time histories of individuals are illustrated in Fig. 6. As can be seen, the maximum number of infected people has decreased when compared to the case where no optimal control effort was made. Also, the decreasing rate of infectious people is noticeably quicker.

\section{Conclusion}

Here, in the present investigation, a attempt has been made to study a new mathematical model for the dynamics of novel coronavirus. We first formulated a new mathematical model for the better understanding of the virus by considering the hospitalized class. After formulation the model, we presented in details the model analysis for the integer-order case and obtained the stability results in detailed. We found the local and global asymptotical stability of the model when $\mathcal{R}_{0}<1$. 
Fig. 6 The number of individuals with optimal control
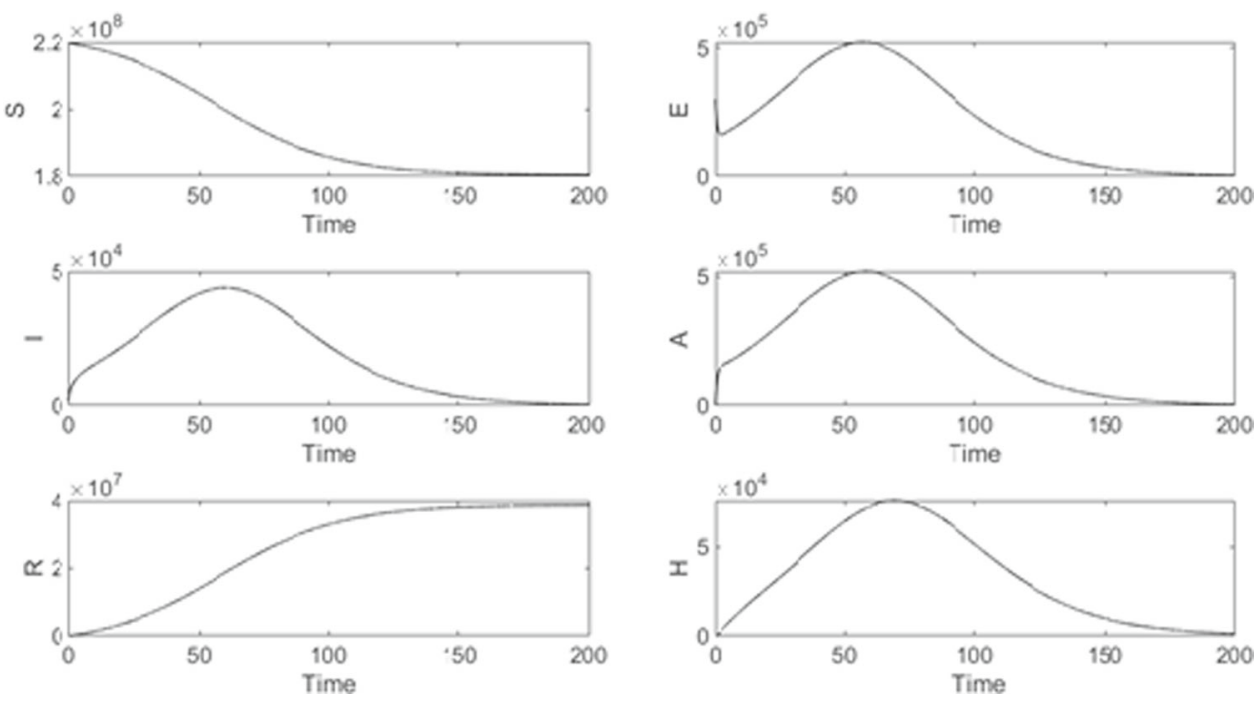

Furthermore, we estimated the parameters using the real cases of coronavirus from Pakistan and obtained the results for the data fitting. The realistic parameters for the considered data gave the basic reproduction number $\mathcal{R}_{0}=1.2036$. Using further parameter values, some graphical results are obtained that indicate the infection can be minimized by following the reduction in contact rate, asymptomatic contact, rapid testing of individuals to isolate the individuals with no clinical symptoms. The numerical results for the integer cases can be useful for the minimization of infection by following the suggestions given in figures in numerical results section. Finally, a variable-order fractional model of SARS-CoV-2 in Pakistan was introduced. Then, through an evolutionary algorithm, the control of the variable-order fraction model was studied. Through numerical simulations, it was shown that using the proposed optimal strategy, the number of infected people has decreased significantly, and the third wave is controlled more efficiently.

Acknowledgements The authors extend their appreciation to the Deanship of Scientific Research at King Khalid University, Abha, Saudi Arabia, for funding this work through research groups program under grant number RGP.1/7/42. This research was supported by the Taif University Researchers Supporting Project number (TURSP2020/77), Taif University, Taif, Saudi Arabia.

Data availibility statement This manuscript has associated data in a data repository. [Authors' comment: The data is available from the corresponding author on a reasonable request.]

\section{Declarations}

Conflicts of interest No conflicts of interest exist regarding the publishing of this work.

\section{References}

1. X.-P. Li, N. Gul, M. A. Khan, R. Bilal, A. Ali, M. Y. Alshahrani, T. Muhammad, S. Islam, A new Hepatitis $\mathrm{B}$ model in light of asymptomatic carriers and vaccination study through Atangana-Baleanu derivative, Results Phys., 2021, 29, Article ID 104603, 10 pages. https://doi.org/10.1016/j.rinp.2021.104603

2. Y.-X. Li, M. H. Alshbool, Y.-P. Lv, I. Khan, M. R. Khan, A. Issakhov, Heat and mass transfer in MHD Williamson nanofluid flow over an exponentially porous stretching surface, Case Stud. Therm. Eng., 2021, 26, Article ID 100975, 10 pages. https://doi.org/10.1016/j. csite. 2021.100975

3. P.-Y. Xiong, M.I. Khan, R.J.P. Gowda, R.N. Kumar, B.C. Prasannakumara, Y.-M. Chu, Comparative analysis of (Zinc ferrite, Nickel Zinc ferrite) hybrid nanofluids slip flow with entropy generation, Modern Phys. Lett. B 35(20), 2150342 (2021) 10 pages. https://doi.org/10. $1142 / \mathrm{S} 0217984921503425$

4. Muhammad Altaf Khan, Abdon Atangana, Modeling the dynamics of novel coronavirus (2019-ncov) with fractional derivative. Alexandria Eng. J. 59(4), 2379-2389 (2020)

5. Leonardo López, Xavier Rodo, A modified seir model to predict the covid-19 outbreak in spain and italy: simulating control scenarios and multi-scale epidemics. Results Phys. 21, 103746 (2021)

6. Denis Efimov and Rosane Ushirobira. On an interval prediction of covid-19 development based on a seir epidemic model. Annual reviews in control, 2021

7. Elena Loli Piccolomini, Fabiana Zama, Monitoring italian covid-19 spread by a forced seird model. PloS One 15(8), e0237417 (2020)

8. Farai Nyabadza, Faraimunashe Chirove, CW Chukwu, and Maria Vivien Visaya. Modelling the potential impact of social distancing on the covid-19 epidemic in south africa. Computational and mathematical methods in medicine, 2020, 2020

9. Aniruddha Adiga, Devdatt Dubhashi, Bryan Lewis, Madhav Marathe, Srinivasan Venkatramanan, and Anil Vullikanti. Mathematical models for covid-19 pandemic: 
a comparative analysis. J Indian Inst Sci, pages 1-15, 2020

10. O. Torrealba-Rodriguez, R.A. Conde-Gutiérrez, A.L. Hernández-Javier, Modeling and prediction of covid-19 in mexico applying mathematical and computational models. Chaos Solitons Fractals 138, 109946 (2020)

11. Yu-Ming Chu, Aatif Ali, Muhammad Altaf Khan, Saeed Islam, and Saif Ullah. Dynamics of fractional order covid-19 model with a case study of saudi arabia. Results Phys, 21:103787, 2021

12. Marei Saeed Alqarni, Metib Alghamdi, Taseer Muhammad, Ali Saleh Alshomrani, and Muhammad Altaf Khan. Mathematical modeling for novel coronavirus (covid-19) and control. Numer Methods Partial Differ Equ, 2020

13. Saif Ullah, Muhammad Altaf Khan, Modeling the impact of non-pharmaceutical interventions on the dynamics of novel coronavirus with optimal control analysis with a case study. Chaos Solitons Fractals 139, 110075 (2020)

14. Abdon Atangana, Modelling the spread of covid-19 with new fractal-fractional operators: Can the lockdown save mankind before vaccination? Chaos, Solitons Fractals 136, 109860 (2020)

15. Joshua Kiddy K Asamoah, Mark A Owusu, Zhen Jin, FT Oduro, Afeez Abidemi, and Esther Opoku Gyasi. Global stability and cost-effectiveness analysis of covid19 considering the impact of the environment: using data from ghana. Chaos Solitons Fractals, 140:110103, 2020

16. M. Higazy, Novel fractional order sidarthe mathematical model of covid-19 pandemic. Chaos Solitons Fractals 138, 110007 (2020)

17. Sümeyye. Çakan, Dynamic analysis of a mathematical model with health care capacity for covid-19 pandemic. Chaos Solitons Fractals 139, 110033 (2020)

18. A, Boudaoui, El hadj Moussa Y, Hammouch Z, Ullah S. A fractional-order model describing the dynamics of the novel coronavirus (COVID-19) with nonsingular kernel. Chaos, Solitons \& Fractals. 2021 May 1;146:110859

19. M. Zamir, F. Nadeem, T. Abdeljawad, Z. Hammouch, Threshold condition and non pharmaceutical interventionss control strategies for elimination of COVID-19. Results Phys. 1(20), 103698 (2021)

20. P. Sahoo, H.S. Mondal, Z. Hammouch, T. Abdeljawad, D. Mishra, M. Reza, On the necessity of proper quarantine without lock down for 2019-nCoV in the absence of vaccine. Results Phys. 1(25), 104063 (2021)

21. H. Jahanshahi, J.M. Munoz-Pacheco, S. Bekiros, N.D. Alotaibi, A fractional-order SIRD model with timedependent memory indexes for encompassing the multifractional characteristics of the COVID-19. Chaos Solitons Fractals 143, 110632 (2021)

22. P. Pandey, Y.M. Chu, J.F. Gómez-Aguilar, H. Jahanshahi, A.A. Aly, A novel fractional mathematical model of COVID-19 epidemic considering quarantine and latent time. Results Phys. 26, 104286 (2021)

23. Ali KK, Osman MS, Baskonus HM, Elazabb NS, Ilhan E. Analytical and numerical study of the HIV-1 infection of CD4+ T-cells conformable fractional mathematical model that causes acquired immunodeficiency syndrome with the effect of antiviral drug therapy. Mathematical Methods in the Applied Sciences. 2020 Nov 5
24. S. Kumar, R. Kumar, M.S. Osman, B. Samet, A wavelet based numerical scheme for fractional order SEIR epidemic of measles by using Genocchi polynomials. Numer Methods Partial Differ Equ. 37(2), 1250-68 (2021)

25. B. Inan, M.S. Osman, T. Ak, D. Baleanu, Analytical and numerical solutions of mathematical biology models: the Newell-Whitehead-Segel and Allen-Cahn equations. Math. Methods Appl. Sci. 43(5), 2588-600 (2020)

26. O.A. Arqub, M.S. Osman, A.H. Abdel-Aty, A.B. Mohamed, S. Momani, A numerical algorithm for the solutions of ABC singular LaneEmden type models arising in astrophysics using reproducing kernel discretization method. Mathematics. 8(6), 923 (2020)

27. Z.-H. Shen, Y.-M. Chu, M. A. Khan, S. Muhammad, O. A. AlHartomy, M. Higazy, Mathematical modeling and optimal control of the COVID-19 dynamics, Results Phy., 2021, 31, Article ID 105026, 9 pages. https://doi. org/10.1016/j.rinp.2021.105028

28. X.-P. Li, H. Al Bayatti, A. Din, A. Zeb, A vigorous study of fractional order COVID-19 model via ABC derivatives, Results Phys., 2021, 29, Article ID 104737, 12 pages. https://doi.org/10.1016/j.rinp.2021.104737

29. B. Wang, H. Jahanshahi, H. Dutta, E. ZambranoSerrano, V. Grebenyuk, S. Bekiros, A.A. Aly, Incorporating fast and intelligent control technique into ecology: a Chebyshev neural network-based terminal sliding mode approach for fractional chaotic ecological systems. Ecol. Complexity 47, 100943 (2021)

30. Z. Liu, H. Jahanshahi, J.F. Gómez-Aguilar, G. Fernandez-Anaya, J. Torres-Jiménez, A.A. Aly, A.M. Aljuaid, Fuzzy adaptive control technique for a new fractional-order supply chain system. Physica Scripta 96(12), 124017 (2021)

31. H. Wang, H. Jahanshahi, M.K. Wang, S. Bekiros, J. Liu, A.A. Aly, A Caputo-Fabrizio fractional-order model of HIV/AIDS with a treatment compartment: sensitivity analysis and optimal control strategies. Entropy 23(5), 610 (2021)

32. Y.L. Wang, H. Jahanshahi, S. Bekiros, F. Bezzina, Y.M. Chu, A.A. Aly, Deep recurrent neural networks with finite-time terminal sliding mode control for a chaotic fractional-order financial system with market confidence. Chaos Solitons Fractals 146, 110881 (2021)

33. Y.M. Chu, S. Bekiros, E. Zambrano-Serrano, O. OrozcoLópez, S. Lahmiri, H. Jahanshahi, A.A. Aly, Artificial macro-economics: a chaotic discrete-time fractionalorder laboratory model. Chaos Solitons Fractals 145, 110776 (2021)

34. P.Y. Xiong, H. Jahanshahi, R. Alcaraz, Y.M. Chu, J.F. Gómez-Aguilar, F.E. Alsaadi, Spectral entropy analysis and synchronization of a multi-stable fractionalorder chaotic system using a novel neural network-based chattering-free sliding mode technique. Chaos Solitons Fractals 144, 110576 (2021)

35. K. Rajagopal, H. Jahanshahi, S. Jafari, R. Weldegiorgis, A. Karthikeyan, P. Duraisamy, Coexisting attractors in a fractional order hydro turbine governing system and fuzzy PID based chaos control. Asian J Control 23(2), 894-907 (2021)

36. Zúñiga-Aguilar, C. J., Gómez-Aguilar, J. F., RomeroUgalde, H. M., Jahanshahi, H., \& Alsaadi, F. E. (2021). Fractal-fractional neuro-adaptive method for system identification. Engineering with Computers, 1-24 
37. B. Wang, H. Jahanshahi, C. Volos, S. Bekiros, M.A. Khan, P. Agarwal, A.A. Aly, A new RBF neural network-based fault-tolerant active control for fractional time-delayed systems. Electronics 10(12), 1501 (2021)

38. B. Wang, H. Jahanshahi, S. Bekiros, Y.M. Chu, J.F. Gomez-Aguilar, F.E. Alsaadi, M.O. Alassafi, Tracking control and stabilization of a fractional financial risk system using novel active finite-time fault-tolerant controls. Fractals 29(6), 2150155-77 (2021)

39. H. Jahanshahi, A. Yousefpour, J.M. Munoz-Pacheco, S. Kacar, V.T. Pham, F.E. Alsaadi, A new fractionalorder hyperchaotic memristor oscillator: dynamic analysis, robust adaptive synchronization, and its application to voice encryption. Appl. Math. Comput. 383, 125310 (2020)

40. G.W. Bohannan, Comments on time-varying fractional order. Nonlinear Dyn. 90(3), 2137-2143 (2017)

41. R. Behinfaraz, M.A. Badamchizadeh, Synchronization of different fractional order chaotic systems with timevarying parameter and orders. ISA Trans. 80, 399-410 (2018)

42. M.H. Khooban, M. Gheisarnejad, N. Vafamand, J. Boudjadar, Electric vehicle power propulsion system control based on time-varying fractional calculus: implementation and experimental results. IEEE Trans. Intell. Vehicles 4(2), 255-264 (2019)

43. Y. Huang, D. Wang, J. Zhang, F. Guo, Controlling and synchronizing a fractional-order chaotic system using stability theory of a time-varying fractional-order system. Plos One 13(3), e0194112 (2018)

44. B. Wang, J. Liu, M.O. Alassafi, F.E. Alsaadi, H. Jahanshahi, S. Bekiros, Intelligent parameter identification and prediction of variable time fractional derivative and application in a symmetric chaotic financial system (Chaos, Solitons \& Fractals, 2021), p. 111590
45. J.F. Li, H. Jahanshahi, S. Kacar, Y.M. Chu, J.F. Gómez-Aguilar, N.D. Alotaibi, K.H. Alharbi, On the variable-order fractional memristor oscillator: Data security applications and synchronization using a type-2 fuzzy disturbance observer-based robust control. Chaos Solitons Fractals 145, 110681 (2021)

46. H. Jahanshahi, S.S. Sajjadi, S. Bekiros, A.A. Aly, On the development of variable-order fractional hyperchaotic economic system with a nonlinear model predictive controller. Chaos Solitons Fractals 144, 110698 (2021)

47. S. Soradi-Zeid, H. Jahanshahi, Yousefpour, A.,\& Bekiros, S., King algorithm: a novel optimization approach based on variable-order fractional calculus with application in chaotic financial systems. Chaos, Solitons \& Fractals 132, 109569 (2020

48. Pauline Van den Driessche, James Watmough, Reproduction numbers and sub-threshold endemic equilibria for compartmental models of disease transmission. Math. Biosci. 180(1-2), 29-48 (2002)

49. World / countries / pakistan, https://www. worldometers.info/coronavirus/country/pakistan/

50. Pakistan population 1950-2020, https:// www.worldometers.info/world-population/ pakistan-population/

51. X.P. Li, Y. Wang, M.A. Khan, M.Y. Alshahrani, T. Muhammad, A dynamical study of SARS-COV-2: a study of third wave. Results Phys. 1(29), 104705 (2021)

52. V. Daftardar-Gejji, Y. Sukale, S. Bhalekar, A new predictor-corrector method for fractional differential equations. Appl. Math. Comput. 244, 158-182 (2014)

53. K. Diethelm, N.J. Ford, A.D. Freed, A predictorcorrector approach for the numerical solution of fractional differential equations. Nonlinear Dyn. 29(1), 3-22 (2002) 